\title{
MicroRNA profiling and bioinformatics analyses reveal the potential roles of microRNAs in chordoma
}

\author{
KANGWU CHEN*, HAO CHEN*, KAI ZHANG, SIWEI SUN, JIANQIANG MO, \\ JIAN LU, ZHONGLAI QIAN and HUILIN YANG
}

\begin{abstract}
Department of Orthopedic Surgery, The First Affiliated Hospital of Soochow University, Suzhou, Jiangsu 215006, P.R. China
\end{abstract}
Received January 12, 2016; Accepted June 2, 2017

DOI: $10.3892 / 01.2017 .6839$

\begin{abstract}
Chordoma is a rare aggressive bone tumor arising from remnants of the notochord, and patients with chordoma have a poor prognosis. However, the unique expression profiles of microRNAs (miRNAs/miRs) and their downstream signaling pathways in chordoma remain incompletely characterized. The aim of the present study was to delineate the global miRNA expression profile and associated signaling networks in chordoma. miRNA profiling was performed on chordoma and fetal notochord tissues. Differentially expressed miRNAs in chordoma were analyzed using microarrays with hierarchical clustering analysis. The target genes of the differentially expressed miRNAs were predicted, and Gene Ontology (GO) and pathway analyses were performed for the intersecting genes. A total of 42 miRNAs were significantly dysregulated in chordoma compared with that in fetal nucleus pulposus tissues. The expression of hsa-miR-21-3p, hsa-miR-150-5p, hsa-miR-1290 and hsa-miR-623 were validated using the reverse transcription-quantitative polymerase chain reaction. On the basis of the intersection predicted by three databases (Targetscan, microRNA.org and PITA), 10,292 potential miRNA targets were identified. Bioinformatic analyses suggested that these dysregulated miRNAs and their predicted targets were functions of signaling pathways in cancer, the mitogen-activated protein kinase signaling pathway, regulation of actin cytoskeleton, focal adhesion and endocytosis. In particular, human (hsa-)miR-185-5p was identified as a crucial miRNA in chordoma development via the Wnt signaling pathway. The results of the present study provide a comprehensive expression and functional profile of
\end{abstract}

Correspondence to: Dr Huilin Yang or Dr Zhonglai Qian, Department of Orthopedic Surgery, The First Affiliated Hospital of Soochow University, 188 Shizi Street, Suzhou, Jiangsu 215006, P.R. China

E-mail: yanghuilin2008@163.com

E-mail: szspine@163.com

${ }^{*}$ Contributed equally

Key words: chordoma, microRNA, microarray, nucleus pulposus, bioinformatics analyses differentially expressed miRNAs associated with chordoma. This profile may serve as a potential tool for biomarker and therapeutic target identification in patients with chordoma.

\section{Introduction}

Chordoma is a rare locally invasive low-grade malignant bone tumor that is considered to originate from remnants of the embryonic notochord (1). Chordoma accounts for between 1 and $4 \%$ of malignant bone tumors with an annual incidence of 0.08/100,000 individuals (2). Complete removal of chordoma is difficult owing to the requirement to preserve adjacent vital structures, the large tumor size and extensive intraoperative blood loss (3). Additionally, chordoma is largely resistant to chemotherapy and radiotherapy (4). Accordingly, local recurrence rates of sacral chordoma were $>40 \%$, and the 5- and 10-year overall survival rates were 70 and $40 \%$ respectively, which leads to a poor quality of life and poor prognosis of patients with chordoma (5). Therefore, there is a clinical requirement for improving therapeutic options for this life-threatening tumor. Currently, there is inadequate understanding of the genetics and molecular biology of chordoma, and information about the potential molecular therapeutic targets is lacking.

MicroRNAs (miRNAs/miRs) are small non-coding regulatory RNA molecules that exert a range of effects on the regulation of gene expression. Dysregulation of miRNAs that target the expression of oncogenes or tumor suppressor genes may therefore influence a number of essential biological functions, including cancer initiation and progression $(6,7)$. The role of miRNAs in chordoma has been studied when compared with muscle tissue or adult nucleus pulposus tissues (8-10). Certain miRNAs are differentially expressed in chordoma and, in particular, miR-1 and miR-31 may have a functional impact on chordoma tumor pathogenesis $(8,9)$. Nevertheless, the unique expression profiles of miRNAs and their downstream signaling pathways in chordoma remain incompletely characterized. Additionally, to the best of our knowledge, it has been demonstrated that chordoma originate from the remnant notochord (11), and notochordal cells disappear by early childhood and are replaced by the nucleus pulposus in the intervertebral discs (12). Therefore, fetal nucleus pulposus tissues were selected as a control in the present study. The aim of the present study was to delineate the global miRNA 
Table I. Clinical and pathological features of patients.

\begin{tabular}{lclccc}
\hline Patient no. & Age, years & Sex & Tumor site & Tumor size, $\mathrm{mm}$ & Surrounding muscle invasion \\
\hline 1 & 40 & Male & S2 & 110 & Yes \\
2 & 66 & Male & S1 & 135 & Yes \\
3 & 48 & Female & S3 & 90 & No \\
4 & 44 & Male & S2 & 105 & Yes \\
5 & 51 & Male & S3 & 95 & Yes \\
6 & 55 & Female & S2 & 115 & Yes \\
7 & 44 & Female & S2 & 120 & No \\
8 & 46 & Female & S3 & 100 & Yes \\
9 & 45 & Male & S4 & 60 & No \\
10 & 50 & Female & S2 & 110 & Yes \\
11 & 37 & Female & S1 & 155 & Yes \\
12 & 60 & Male & S3 & 85 & No \\
13 & 38 & Female & S2 & 110 & Yes \\
14 & 22 & Male & S1 & 130 & Yes \\
15 & 56 & Male & S2 & 115 & No \\
\hline
\end{tabular}

expression profile and associated signaling networks in chordoma.

\section{Materials and methods}

Tissue samples. A total of 15 chordoma tissues from patients who had undergone surgical resection of chordoma were obtained from the Department of Orthopedic Surgery, The First Affiliated Hospital of Soochow University (Suzhou, China) between January 2005 and June 2015. The clinical and pathological features of the patients are listed in Table I. A total of 10 fetal nucleus pulposus tissues from the intervertebral discs of aborted fetuses between the 20 and 28th week of gestation were included as a control group. Fresh specimens were collected immediately following resection, snap-frozen in liquid nitrogen and stored at $-80^{\circ} \mathrm{C}$ until total RNA extraction. Samples were taken from areas exhibiting neither hemorrhage nor necrosis. These samples were confirmed by two pathologists. The present study was approved by the Ethics Committee of The First Affiliated Hospital of Soochow University and written informed consent was provided by all patients.

RNA isolation and miRNA microarray profiling. Total RNA was extracted from three chordoma and three fetal nucleus pulposus fresh-frozen tissue samples using mirVana ${ }^{\mathrm{TM}}$ RNA Isolation Kit (Applied Biosystems; Thermo Fisher Scientific, Inc., Waltham, MA, USA), according to the manufacturer's protocol. Total RNA was quantified using a NanoDrop ND-2000 instrument (Thermo Fisher Scientific, Inc.) and RNA integrity was assessed using an Agilent Bioanalyzer 2100 instrument (Agilent Technologies, Inc., Santa Clara, CA, USA). RNAs with a 2100RIN (RNA integrity number) 6.0 and $28 \mathrm{~S} / 18 \mathrm{~S}$ ratio 0.7 were used for the miRNA array analysis and reverse transcription (RT).

miRNA expression profiling was performed using Agilent Human miRNA Microarray kit (Agilent Technologies, Inc.) containing 2,006 human mature miRNAs. Total RNAs were dephosphorylated, denaturized and then labeled with cyanine-3-cytosine triphosphate. Following purification, the labeled RNAs were hybridized onto the microarray. Following washing, the arrays were scanned using an Agilent Scanner G2505C (Agilent Technologies, Inc.). Genespring software (version 12.5, Agilent Technologies, Inc.) was used for microarray analysis, with the raw data normalized using the quantile algorithm. Differentially expressed miRNAs were identified through fold changes as well as P-values calculated using a Student's t-test. The thresholds set for up- and downregulated genes were a fold change $\geq 2.0$ and $\mathrm{P} \leq 0.05$.

Bioinformatics analysis. Hierarchical clustering was performed to identify the distinguishable miRNA expression pattern among samples. Potential target genes of differentially expressed miRNAs were predicted by the intersection of three databases (Targetscan, http://www.targetscan.org; microRNA. org, http://www.microrna.org; and PITA, http://genie .weizmann.ac.il/pubs/mir07/mir07_dyn_data.html). For functional analyses of potential miRNA targets, Gene Ontology (GO) term analysis was applied to organize genes into categories on the basis of biological processes, cellular components and molecular functions. Biological pathways analysis, which was defined by the Kyoto Encyclopedia of Genes and Genomes (KEGG; www.genome.ad.jp/kegg), provided an improved understanding of gene expression information as a complete network. Fisher's exact test, $\chi^{2}$ test, and the threshold of significance were defined by the P-value and false discovery rate (FDR). The screening criterion was $\mathrm{P}<0.05$.

$R T$-quantitative polymerase chain reaction ( $q P C R)$. RT-qPCR was used to measure miRNA expression using TaqMan MicroRNA assays (Applied Biosystems; Thermo Fisher Scientific, Inc.). Primer sequences used were as follows: 
miR-21 forward, 5'-TAGCTTATCAGACTGATGTTGA-3' and reverse, 5'-TCA ACATCAGTCTGATAAGCTA-3'; miR-150 forward 5'-CAGTAT TCTCTCCCA ACCCTT GTA-3' and reverse, 5'-AATGGATGATCTCGTCAGTCT GTT-3'; miR-1290 forward, 5'-CAGTGCTGGATTTTT GGAT-3' and reverse, 5'-TATGGTTGTTCACGACTCCTT CAC-3'; miR-623 forward, 5'-CAGAAGCGTAATGGACCT TTCG-3' and reverse, 5'-TATCGCTGATCACGACTCGTT GAT-3'; and U6 forward, 5'-ATTGGAACGATACAGAGA AGATT-3' and reverse, 5'-GGAACGCTTCACGAATTTG-3'. miRNA-specific reverse transcription was performed with 5 ng total RNA using the TaqMan MicroRNA reverse transcription kit (Applied Biosystems; Thermo Fisher Scientific, Inc.), according to the manufacturer's protocol. qPCR was performed using the TaqMan MicroRNA assay kit on the Step-One Plus Real-Time PCR System (Applied Biosystems; Thermo Fisher Scientific, Inc.). Thermal cycling conditions were set according to the manufacturer's fast protocol and all reactions were performed in triplicate. Relative expression was determined using the $2^{-\Delta \Delta \mathrm{Cq}}$ method (13), and expression values were normalized to U6, which has been demonstrated to be a suitable reference gene.

\section{Results}

miRNA array analysis. Three primary chordoma and three fetal nucleus pulposus fresh-frozen tissue samples were selected for the identification of differentially expressed miRNAs. In total, 42 of the 2006 miRNAs analyzed were significantly dysregulated in the chordoma group compared with normal controls at a level of $\mathrm{P}<0.05$ and a fold change $>2$. In total, 23 downregulated and 19 upregulated known miRNAs were identified in chordoma tissues compared with fetal nucleus pulposus tissues (Table II). A non-supervised 2D-cluster analysis was applied for all tumors and the 42 significantly dysregulated miRNAs (Fig. 1).

$G O$ analysis. To further determine the biological functions of these miRNAs, intersection predicted with three databases (Targetscan, microRNA.org and PITA) was used to predict the target genes of the 42 miRNAs, which resulted in the identification of 10,292 putative target genes. The functions of these target genes were then determined using GO analysis. According to the results, 496, 151 and 152 GO terms were identified from three ontologies including biological processes, cellular components and molecular functions, respectively. Significant GO terms corresponding to biological processes included regulation of transcription (DNA-dependent), signal transduction and multicellular organismal development. The main GO terms for cellular components included nucleus, cytoplasm and cytosol. The significant GO categories corresponding to molecular functions included protein binding, metal ion binding and zinc ion binding (Fig. 2A-C). These functions are known to be markedly associated with tumor generation and progress.

KEGG pathway analysis. To achieve an improved understanding of the functions and regulatory networks of predicted target genes, target enrichment was searched for using KEGG. There were 1,805 differentially expressed genes identified
Table II. Significantly dysregulated miRNAs in the chordoma tissues vs. fetal nucleus pulposus tissues.

\begin{tabular}{|c|c|c|c|}
\hline & Fold change & P-value & Regulation \\
\hline hsa-miR-150-5p & 341.1334 & 0.0001 & Up \\
\hline hsa-miR-497-5p & 321.3715 & 0.0042 & Up \\
\hline hsa-miR-21-3p & 240.6141 & $<0.0001$ & $\mathrm{Up}$ \\
\hline hsa-miR-29b-3p & 226.4459 & 0.0205 & $\mathrm{Up}$ \\
\hline hsa-miR-223-3p & 188.2892 & 0.0202 & Up \\
\hline hsa-miR-143-3p & 117.0034 & 0.0011 & Up \\
\hline hsa-miR-222-3p & 78.3672 & 0.0155 & Up \\
\hline hsa-miR-221-3p & 76.1249 & 0.0164 & Up \\
\hline hsa-miR-4261 & 66.7500 & 0.0320 & $\mathrm{Up}$ \\
\hline hsa-miR-374b-5p & 46.1905 & 0.0136 & Up \\
\hline hsa-miR-185-5p & 45.5516 & 0.0222 & Up \\
\hline hsa-miR-93-5p & 44.2137 & 0.0399 & Up \\
\hline hsa-miR-770-5p & 24.4578 & 0.0183 & Up \\
\hline hsa-miR-1260a & 19.4710 & 0.0406 & Up \\
\hline hsa-miR-4485 & 6.2146 & 0.0192 & Up \\
\hline hsa-miR-1233-1-5p & 3.5351 & 0.0064 & Up \\
\hline hsa-miR-365a-3p & 3.4641 & 0.0033 & Up \\
\hline hsa-miR-211-3p & 2.7623 & 0.0052 & Up \\
\hline hsa-miR-193a-5p & 2.2088 & 0.0049 & Up \\
\hline hsa-miR-5196-5p & 244.3847 & 0.0001 & Down \\
\hline hsa-miR-4433-3p & 146.8665 & 0.0452 & Down \\
\hline hsa-miR-4754 & 130.6493 & $<0.0001$ & Down \\
\hline hsa-miR-4707-5p & 130.4238 & 0.0001 & Down \\
\hline hsa-miR-3156-5p & 108.7725 & 0.0173 & Down \\
\hline hsa-miR-3652 & 33.9637 & 0.0467 & Down \\
\hline hsa-miR-1290 & 33.7951 & 0.0455 & Down \\
\hline hsa-miR-3137 & 29.5932 & 0.0158 & Down \\
\hline hsa-miR-623 & 26.7450 & 0.0368 & Down \\
\hline hsa-miR-3917 & 25.9233 & 0.0422 & Down \\
\hline hsa-miR-4656 & 25.3885 & 0.0245 & Down \\
\hline hsa-miR-4710 & 25.1405 & 0.0253 & Down \\
\hline hsa-miR-33b-3p & 15.6259 & 0.0085 & Down \\
\hline hsa-miR-4685-5p & 14.0308 & 0.0302 & Down \\
\hline hsa-miR-4690-5p & 13.1652 & 0.0015 & Down \\
\hline hsa-miR-9-3p & 12.2306 & 0.0286 & Down \\
\hline hsa-miR-1471 & 11.6128 & 0.0325 & Down \\
\hline hsa-miR-1273f & 9.7157 & 0.0105 & Down \\
\hline hsa-miR-1229-5p & 7.1305 & 0.0399 & Down \\
\hline hsa-miR-1246 & 4.7649 & 0.0071 & Down \\
\hline hsa-miR-6127 & 3.7462 & 0.0391 & Down \\
\hline hsa-miR-320e & 2.9118 & 0.0438 & Down \\
\hline hsa-miR-320d & 2.6649 & 0.0459 & Down \\
\hline
\end{tabular}

hsa, human (Homo sapiens).

in 65 pathways with a threshold of $\mathrm{P}<0.01$ and $\mathrm{FDR}<0.05$ using KEGG pathway analysis. Overall, a genetic cluster summarizing the functions of signaling pathways in cancer, mitogen-activated protein kinase (MAPK) signaling pathway, regulation of actin cytoskeleton, focal adhesion and endocytosis 


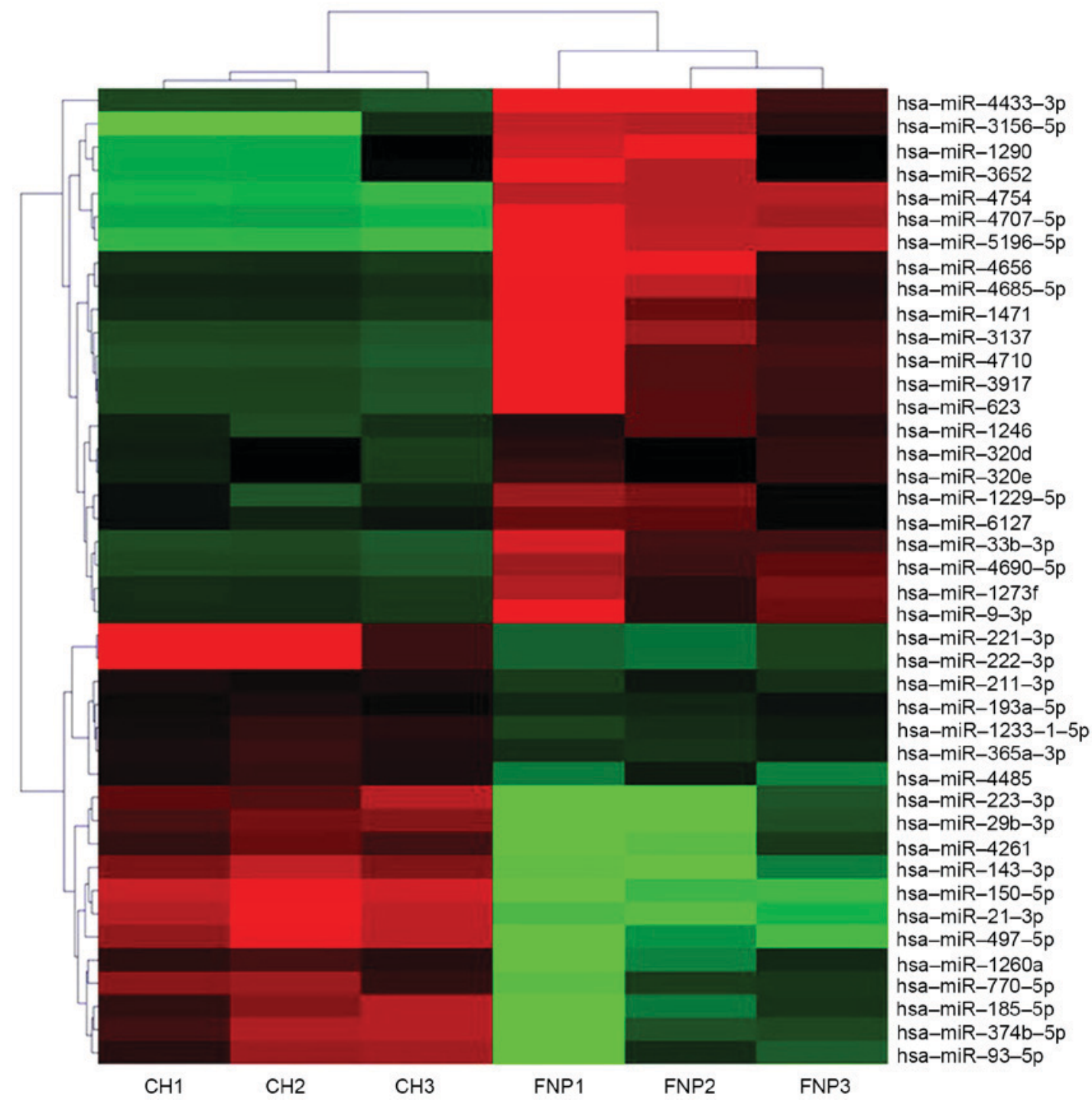

Figure 1. Hierarchical clustering of differentially expressed miRNA genes in chordoma tissues compared with fetal nucleus pulposus tissues. Average linkage clustering was performed using an uncensored correlation metric. Red denotes high expression levels, whereas green depicts low expression levels.CH chordoma samples, FNP fetal nucleus pulposus samples. hsa, human (Homo sapiens); miR, microRNA.

was identified to exhibit the most marked association with the chordoma group (Fig. 3). Notably, the Wnt signaling pathway was dysregulated in chordoma; aberrant Wnt signaling is associated with tumorigenesis in a number of types of tumor.

Validation of miRNA array data. To validate miRNA microarray data, RT-qPCR was performed using TaqMan MicroRNA assays. In total, four miRNAs (hsa-miR-21-3p, hsa-miR-150-5p, hsa-miR-1290 and hsa-miR-623) were randomly selected for validation from the 42 significantly dysregulated miRNAs. As presented in Fig. 4, the expression levels of hsa-miR-21-3p and hsa-miR-150-5p were significantly increased in chordoma tissues compared with in the control group, whereas expression levels of hsa-miR-1290 and hsa-miR-623 were significantly decreased in chordoma tissues compared with the control group. These four miRNAs may therefore serve a role in the malignant progression of chordoma.

Additionally, due to the Wnt signaling pathway potentially serving a pivotal role in chordoma, hsa-miR-185-5p was included for validation which had the potential to target the Wnt signaling pathway. It was confirmed that the expression of hsa-miR-185-5p was dysregulated in chordoma tissues (Fig. 5).

\section{Discussion}

Chordoma is a malignant bone tumor known to arise from the embryonic remnants of the notochord (1). However, the biological features of chordoma remain largely unknown. miRNAs have emerged as key regulators in numerous oncogenic processes. A number of miRNAs have been identified to be involved in the initiation and progression of several types of human cancer $(6,7)$. Therefore, it is relevant to delineate the expression profiles, functions and potential regulatory mechanisms of miRNAs in chordoma. In the present study, the expression signature of miRNAs in chordoma was investigated, and their functions, downstream targets and signaling pathways were analyzed.

First, miRNA expression profiles were described in chordoma. Compared with fetal nucleus pulposus tissues, 
A

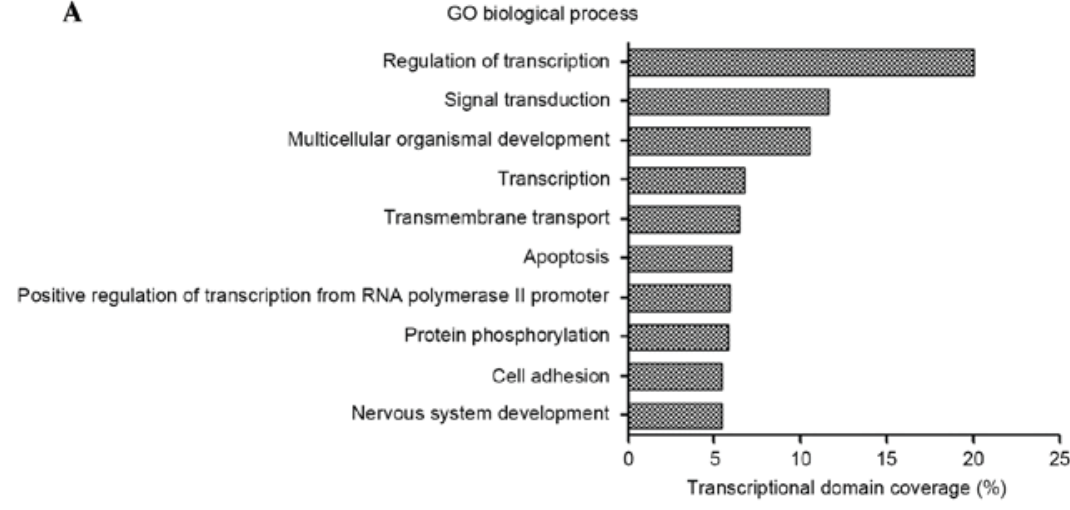

B
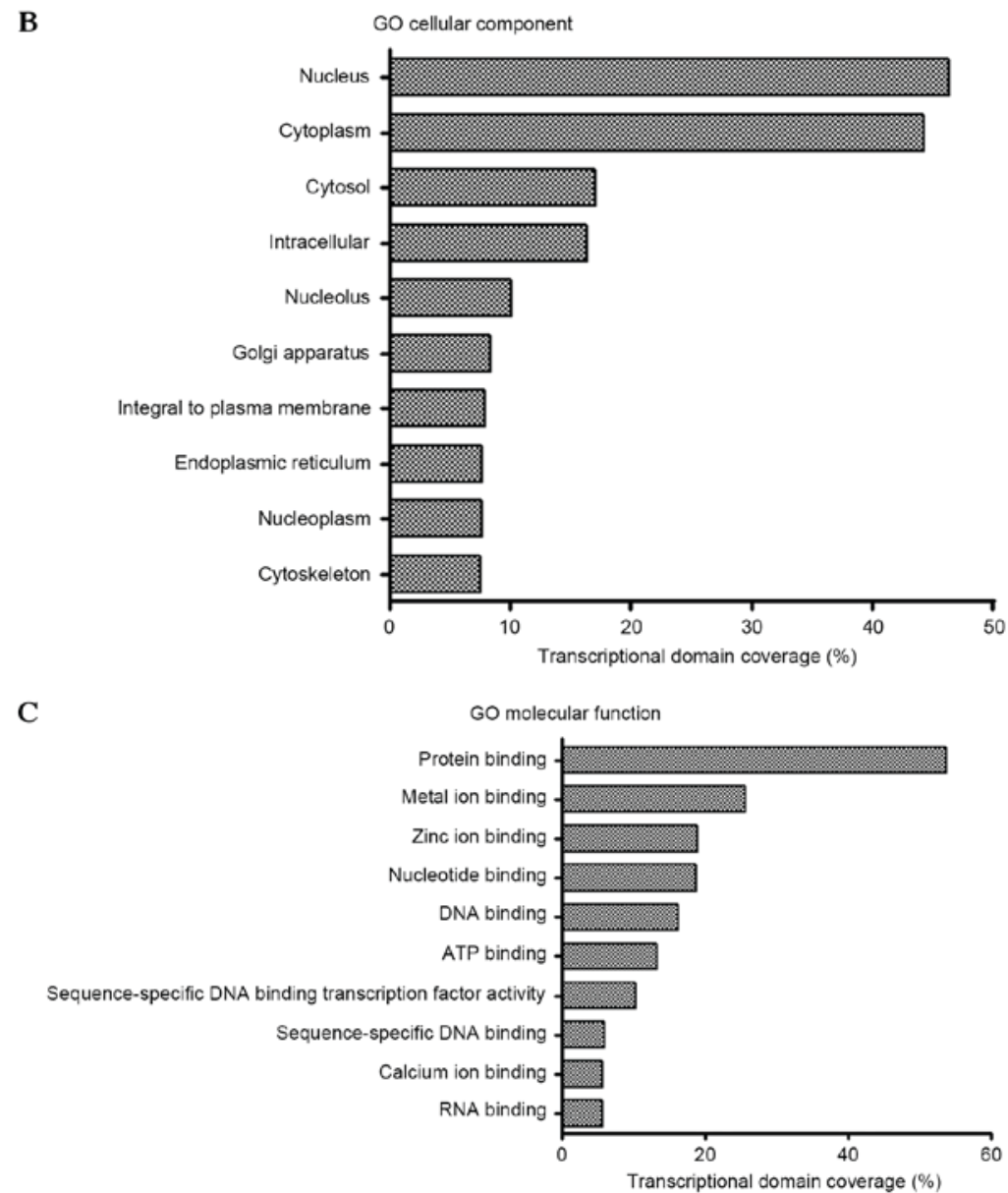

Figure 2. The ten most significant GO terms identified from three ontologisms: (A) Biological processes, (B) cellular components and (C) molecular functions. GO, Gene Ontology; ATP, adenosine 5'-triphosphate.

42 miRNAs were significantly dysregulated in the chordoma group. The differential expression of hsa-miR-21-3p, hsa-miR-150-5p, hsa-miR-1290 and hsa-miR-623 was validated using RT-qPCR. Previously, hsa-miR-21-3p was demonstrated to exhibit oncogenic functions in osteosarcoma, breast cancer and colorectal cancer (14-16). The results of the present study demonstrated that hsa-miR-21-3p expression was increased in chordoma and may regulate invasion and metastasis, and hsa-miR-150-5p is overexpressed in chordoma tissues which acts as an oncogene. Furthermore, hsa-miR-1290 and hsa-miR-623 were downregulated in chordoma tissues. The results suggest that the tumorigenesis of chordoma is characterized by marked changes in miRNA expression profiles.
A number of dysregulated miRNAs that were identified in the present study are reported for the first time in chordoma. Therefore, the results of the present study provide a rationale for further study of these novel dysregulated miRNAs in the initiation and progression of chordoma.

Since the majority of miRNAs are considered to be involved in carcinogenesis by inhibiting target mRNAs, targets of these 42 altered miRNAs were predicted in the present study. In total, 10,292 putative target genes were predicted by the intersection of three databases (Targetscan, microRNA.org and PITA). Further GO analyses revealed that the majority of predicted target genes functioned in regulation of transcription, signal transduction and multicellular organismal development. These 


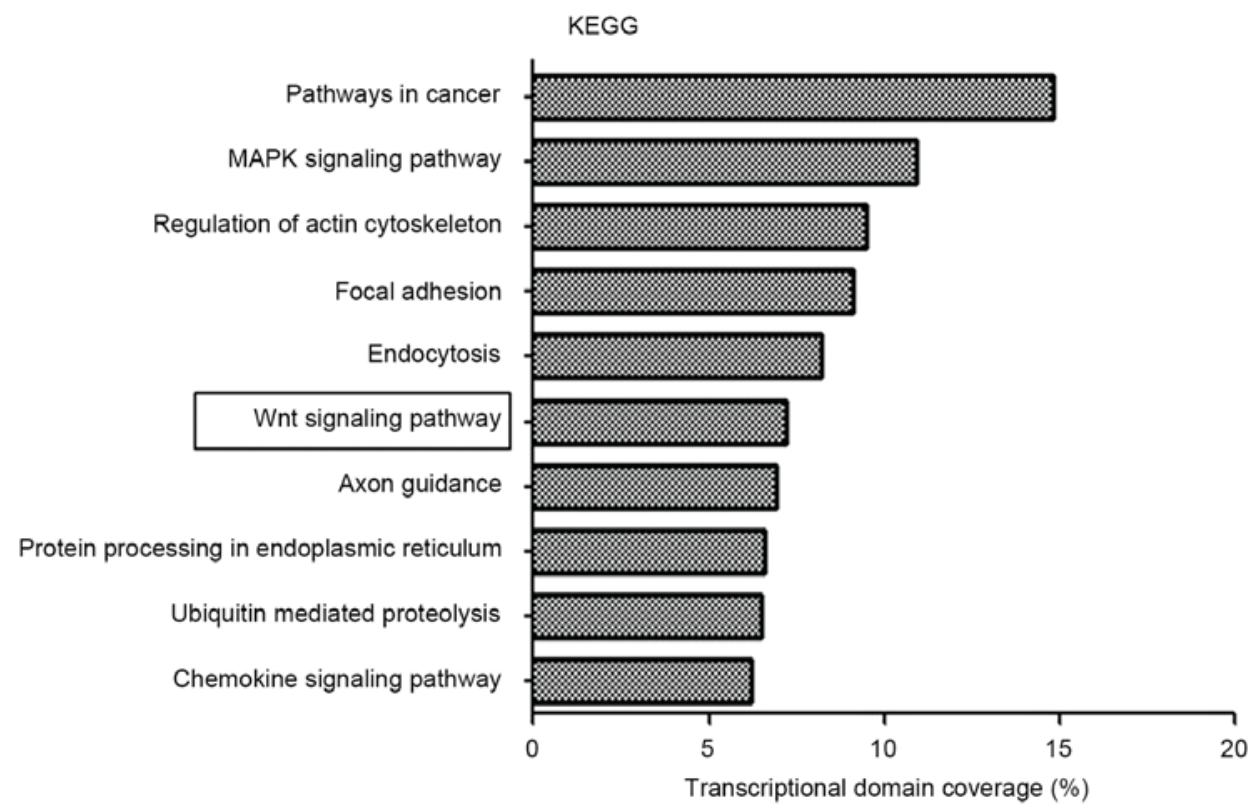

Figure 3. KEGG pathway analysis based on miRNA-targeted genes. KEGG, Kyoto Encyclopedia of Genes and Genomes; MAPK, mitogen-activated protein kinase.

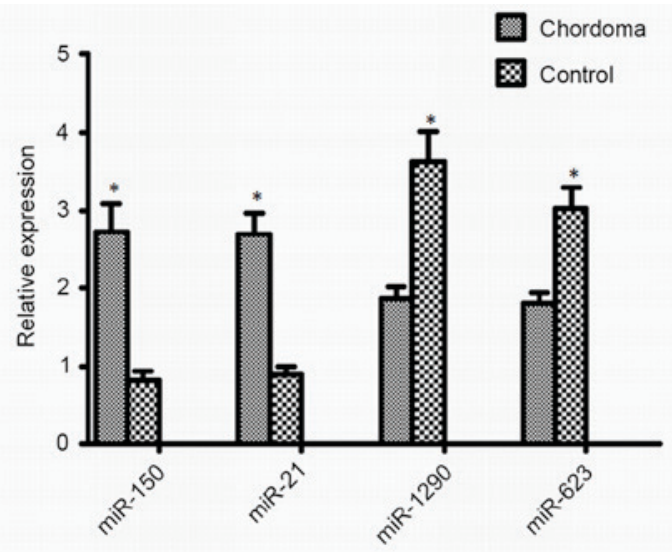

Figure 4. Validation of miRNA expression in chordoma. Differentially expressed miRNAs (miR-21, miR-150, miR-1290 and miR-623) in chordoma $(\mathrm{n}=15)$ relative to fetal nucleus pulposus samples $(\mathrm{n}=10)$. ${ }^{*} \mathrm{P}<0.05$. miR, microRNA.

functions are known to be associated with human tumorigenesis and cancer progression.

Signaling pathways regulated by validated targets of the dysregulated miRNAs were assessed using KEGG pathway analysis. In the present study, the most significant pathways were associated with tumor pathogenesis, including signaling pathways in cancer, MAPK signaling pathway, regulation of actin cytoskeleton, focal adhesion and endocytosis. Consistent with previous studies, the MAPK signaling pathway was the most highly overrepresented genetic pathway $(17,18)$. Additionally, our previous study demonstrated that Raf-1 and extracellular-signal-regulated kinase $1 / 2$, which are important members of the MAPK signaling pathway, were overexpressed in chordoma and associated with tumor progression (19). However, further study was required to evaluate the exact role of the MAPK signaling pathway in the development and progression of chordoma.

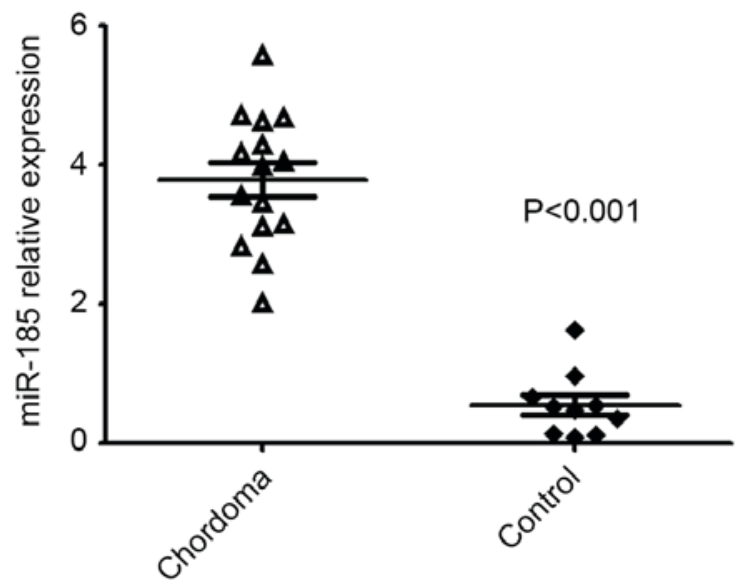

Figure 5. miR-185 is overexpressed in chordoma samples $(\mathrm{n}=15)$ compared with fetal nucleus pulposus samples $(\mathrm{n}=10)$. miR, microRNA.

In particular, the Wnt signaling pathway was demonstrated to be important in chordoma development. Previous studies have indicated that the Wnt signaling pathway serves an important role in cell proliferation, differentiation, survival and apoptosis, and is implicated in various tumor types $(20,21)$. In the present study, it was observed that hsa-miR-185-5p was significantly dysregulated in chordoma tissues and had the potential to target the Wnt signaling pathway. Li et al (22) confirmed that miR-185-3p contributed to the radioresistance of nasopharyngeal carcinoma via modulation of WNT2B expression in vitro. Liu et al (23) confirmed that miR-185 was a negative regulator of RhoA and cell division cycle 42 , and their cellular activities, and was able to inhibit proliferation and invasion of colorectal cancer cells. Fu et al (24) demonstrated that miR-185 inhibited the proliferation of breast cancer cells by regulating the expression of c-Met, indicating its potential as a therapeutic 
target for breast cancer. Therefore, elucidating oncogenic mechanisms by which miRNAs regulate the Wnt signaling pathway represents a promising strategy for identifying novel therapeutic targets in chordoma.

In conclusion, 42 significantly dysregulated miRNAs were discovered in chordoma, including 19 upregulated and 23 downregulated miRNAs. In addition, 10,292 potential target transcripts were predicted for these 42 miRNAs. Integrated GO and KEGG pathway analyses indicated that the MAPK signaling pathway may serve an important role in chordoma development. In particular, the Wnt signaling pathway may have a vital functional effect on chordoma tumor pathogenesis, and hsa-miR-185-5p was revealed as a potential key oncogenic miRNA in chordoma development via the Wnt signaling pathway. These results indicate that dysregulated expression of miRNAs and their potential target mRNAs are prominently involved in the pathogenesis of chordoma, which suggests that dysregulated miRNAs may serve as potential biomarkers and therapeutic targets in patients with chordoma.

\section{Acknowledgements}

The present study was funded by the Jiangsu Provincial Special Program of Medical Science (grant no. BL2012004), the Natural Science Foundation of the Colleges and Universities in Jiangsu Province (grant no. 16KJD320004) and the Suzhou Civic 'Science and Education Guardian' Youth Science and Technology Project (grant no. KJXW2014009).

\section{References}

1. Casali PG, Stacchiotti S, Sangalli C, Olmi P and Gronchi A: Chordoma. Curr Opin Oncol 19: 367-370, 2007.

2. McMaster ML, Goldstein AM, Bromley CM, Ishibe N and Parry DM: Chordoma: Incidence and survival patterns in the United States, 1973-1995. Cancer Causes Control 12: 1-11, 2001.

3. Fuchs B, Dickey ID, Yaszemski MJ, Inwards CY and Sim FH: Operative management of sacral chordoma. J Bone Joint Surg Am 87: 2211-2216, 2005.

4. Sciubba DM, Cheng JJ, Petteys RJ, Weber KL, Frassica DA and Gokaslan ZL: Chordoma of the sacrum and vertebral bodies. J Am Acad Orthop Surg 17: 708-717, 2009.

5. Chugh R, Tawbi H, Lucas DR, Biermann JS, Schuetze SM and Baker LH: Chordoma: The nonsarcoma primary bone tumor. Oncologist 12: 1344-1350, 2007.

6. Lujambio A and Lowe SW: The microcosmos of cancer. Nature 482: 347-355, 2012.

7. Kong YW, Ferland-McCollough D, Jackson TJ and Bushell M: microRNAs in cancer management. Lancet Oncol 13: e249-e258, 2012.
8. Duan Z, Choy E, Nielsen GP, Rosenberg A, Iafrate J, Yang C, Schwab J, Mankin H, Xavier R and Hornicek FJ: Differential expression of microRNA (miRNA) in chordoma reveals a role for miRNA-1 in Met expression. J Orthop Res 28: 746-752, 2010.

9. Bayrak OF, Gulluoglu S, Aydemir E, Ture U, Acar H, Atalay B, Demir Z, Sevli S, Creighton CJ, Ittmann M, et al: MicroRNA expression profiling reveals the potential function of microRNA-31 in chordomas. J Neurooncol 115: 143-151, 2013.

10. Zhang Y, Schiff D, Park D and Abounader R: MicroRNA-608 and microRNA-34a regulate chordoma malignancy by targeting EGFR, Bcl-xL and MET. PLoS One 9: e91546, 2014.

11. Vujovic S, Henderson S, Presneau N, Odell E, Jacques TS, Tirabosco R, Boshoff C and Flanagan AM: Brachyury, a crucial regulator of notochordal development, is a novel biomarker for chordomas. J Pathol 209: 157-165, 2006.

12. Shen J, Shi Q, Lu J, Wang DL, Zou TM, Yang HL and Zhu GQ: Histological study of chordomaorigin from fetal notochordal cell rests. Spine (Phila Pa 1976) 38: 2165-2170, 2013.

13. Livak KJ and Schmittgen TD: Analysis of relative gene expression data using real-time quantitative PCR and the 2(-Delta Delta C(T)) method. Methods 25: 402-408, 2001.

14. Ziyan W, Shuhua Y, Xiufang W and Xiaoyun L: MicroRNA-21 is involved in osteosarcoma cell invasion and migration. Med Oncol 28: 1469-1474, 2011.

15. Zhang C, Liu K, Li T, Fang J, Ding Y, Sun L, Tu T, Jiang X, Du S, Hu J, et al: miR-21: A gene of dual regulation in breast cancer. Int J Oncol 48: 161-172, 2016.

16. Basati G, Emami Razavi A, Abdi S and Mirzaei A: Elevated level of microRNA-21 in the serum of patients with colorectal cancer. Med Oncol 31: 205, 2014.

17. Long C, Jiang L, Wei F, Ma C, Zhou H, Yang S, Liu X and Liu Z: Integrated miRNA-mRNA analysis revealing the potential roles of miRNAs in chordomas. PLoS One 8: e66676, 2013.

18. Tamborini E, Virdis E, Negri T, Orsenigo M, Brich S, Conca E, Gronchi A, Stacchiotti S, Manenti G, Casali PG, et al: Analysis of receptor tyrosine kinases (RTKs) and downstream pathways in chordomas. Neuro Oncol 12: 776-789, 2010.

19. Zhang K, Chen H, Zhang B, Sun J, Lu J, Chen K and Yang H: Overexpression of Raf-1 and ERK1/2 in sacral chordoma and association with tumor recurrence. Int J Clin Exp Pathol 8: 608-614, 2015.

20. Tian J, He H and Lei G: Wnt/ $/$-catenin pathway in bone cancers. Tumour Biol 35: 9439-9445, 2014.

21. Song JL, Nigam P, Tektas SS and Selva E: microRNA regulation of Wnt signaling pathways in development and disease. Cell Signal 27: 1380-1391, 2015.

22. Li G, Wang Y, Liu Y, Su Z, Liu C, Ren S, Deng T, Huang D, Tian Y and Qiu Y: miR-185-3p regulates nasopharyngeal carcinoma radioresistance by targeting WNT2B in vitro. Cancer Sci 105: 1560-1568, 2014.

23. Liu M, Lang N, Chen X, Tang Q, Liu S, Huang J, Zheng Y and Bi F: miR-185 targets RhoA and Cdc42 expression and inhibits the proliferation potential of human colorectal cells. Cancer Lett 301: 151-160, 2011.

24. Fu P, Du F, Yao M, Lv K and Liu Y: MicroRNA-185 inhibits proliferation by targeting c-Met in human breast cancer cells. Exp Ther Med 8: 1879-1883, 2014. 